PROCEEDINGS OF THE

AMERICAN MATHEMATICAL SOCIETY

Volume 132, Number 7, Pages 1937-1941

S 0002-9939(04)07379-4

Article electronically published on January 30, 2004

\title{
MUMFORD CURVES WITH MAXIMAL AUTOMORPHISM GROUP
}

\author{
GUNTHER CORNELISSEN AND FUMIHARU KATO
}

(Communicated by Michael Stillman)

\begin{abstract}
A Mumford curve of genus $g \notin\{0,1,5,6,7,8\}$ over a non-Archimedean valued field of characteristic $p>0$ has at most $2 \sqrt{g}(\sqrt{g}+1)^{2}$ automorphisms. In this note, the unique family of curves that attains this bound, and its automorphism group, are determined.
\end{abstract}

\section{INTRODUCTION}

It is well known (cf. Conder [2]) that if a compact Riemann surface of genus $g \geq 2$ attains Hurwitz's bound $84(g-1)$ on its number of automorphisms, then its automorphism group is a so-called Hurwitz group, i.e., a finite quotient of the triangle group $\Delta(2,3,7)$. Equivalently, the Riemann surface is an étale cover of the Klein quartic $X(7)$. However, it is hitherto unknown which finite groups can occur as Hurwitz groups. Observe that for every integer $n$ there exists a $g$ such that there are more than $n$ non-isomorphic Riemann surfaces of genus $g$ that attain Hurwitz's bound (Cohen, [1]). In this note, we want to show that the corresponding questions for Mumford curves of genus $g \geq 2$ over non-Archimedean valued fields of positive characteristic have a very easy answer: the maximal automorphism groups can be explicitly described, and they occur for an explicitly given 1-parameter family of curves (at least for $g \notin\{5,6,7,8\}$ ).

The set-up for our result is as follows. Let $(k,|\cdot|)$ be a non-Archimedean valued field of positive characteristic $p>0$, and $X$ a Mumford curve ([8], [6]) of genus $g$ over $k$. This means that the stable reduction of $X$ over the residue field $\bar{k}$ of $k$ is a union of rational curves intersecting in $\bar{k}$-rational points. Equivalently, as a rigid analytic space over $k$, the analytification $X^{\text {an }}$ of $X$ is isomorphic to an analytic space of the form $\Gamma \backslash\left(\mathbf{P}_{k}^{1, \text { an }}-\mathcal{L}\right)$, where $\Gamma$ (the so-called Schottky group of $X$ ) is a discrete free subgroup of $P G L(2, k)$ of rank $g$ (acting in the obvious way on $\mathbf{P}_{k}^{1, \text { an }}$ ) with $\mathcal{L}$ as its set of limit points.

In [4, it was shown that a Mumford curve of genus $g \geq 2$ satisfies

$$
|\operatorname{Aut}(X)| \leq \max \{12(g-1), F(g)\} \text {, where } F(g):=2 \sqrt{g}(\sqrt{g}+1)^{2} .
$$

Received by the editors December 21, 2000 and, in revised form, April 18, 2003.

2000 Mathematics Subject Classification. Primary 14H37, 14G22.

This work was done when the first author was visiting Kyoto University. The main result of this paper answers positively a question posed by T. Sekiguchi during the 2000 Kinosaki Symposium on Algebraic Geometry. 
Remark 1. Note that $F(g)<12(g-1)$ precisely when $g \in\{5,6,7,8\}$. For such $g$, all curves with 12(g-1) automorphisms were found in [5].

For $g \notin\{5,6,7,8\}$, it was shown in 4 that the so-called Artin-Schreier-Mumford curves $X_{t, c}$ attain the bound (1). Here, $t$ is a nonnegative integer, $c \in k^{*}$ satisfies $|c|<1$, and the affine equation of $X_{t, c}$ is given by

$$
X_{t, c}:\left(y^{p^{t}}-y\right)\left(x^{p^{t}}-x\right)=c .
$$

The genus of $X_{t, c}$ is $g_{t}:=\left(p^{t}-1\right)^{2}$, and its automorphism group is isomorphic to

$$
A_{t}:=\mathbf{Z}_{p}^{2 t} \rtimes D_{p^{t}-1}
$$

where $\mathbf{Z}_{n}$ denotes the cyclic group of order $n$, and $D_{n}=\mathbf{Z}_{n} \rtimes \mathbf{Z}_{2}$ is the dihedral group of order $2 n$. The action of the automorphisms on the coordinates is explicitly given by interchanging $x$ and $y$, adding an element of $\mathbf{F}_{p^{t}}$ to $x$ or $y$, and multiplying $x$ and $y$ by $a \in \mathbf{F}_{p^{t}}^{*}$ and $a^{-1}$ respectively. For fixed $t$ and varying $c$, the family $X_{t, c}$ over the punctured unit disc $\Delta^{*}:=\{0<|c|<1\}$ is non-constant (since it extends to a semistable family over the unit disk with a singular fiber at $c=0$ ). Actually, the curve $X_{t, c}$ is exactly Mumford if $|c|<1$, since otherwise, its reduction is irreducible over $\bar{k}$. The aim of this paper is to show the following:

Theorem. Let $g \notin\{0,1,5,6,7,8\}$. Every Mumford curve over a non-Archimedean valued field $k$ of positive characteristic with the maximal number $F(g)$ of automorphisms is isomorphic to an Artin-Schreier-Mumford curve, i.e., an element of the family $\left\{X_{t, c}\right\}_{c \in \Delta^{*}}$ for $t=\log _{p}(\sqrt{g}+1)$; hence its automorphism group is of the form $A_{t}$.

\section{Proof of the theorem}

Let $X$ be a Mumford curve of genus $g$ with $F(g)$ automorphisms. Observe that $\operatorname{Aut}(X)=N / \Gamma$, where $\Gamma$ is the Schottky group of $X$ and $N$ is its normalizer in $P G L(2, k)$ ([6], VII.1). Set $A:=\operatorname{Aut}(X)$, and recall that the only information given about $A$ is that its order is $2 \sqrt{g}(\sqrt{g}+1)^{2}$, where $g$ is the rank of $\Gamma$. Let $-: N \rightarrow A$ denote the reduction map modulo $\Gamma$. For integers $t, n$ such that $n \mid p^{t}-1$, let $B(t, n)=\mathbf{Z}_{p}^{t} \rtimes \mathbf{Z}_{n}$, where the semi-direct action is that of the Borel subgroup of $P G L(2, k)$.

The first part of the proof consists in showing the following, which is the (much easier) non-Archimedean analogue of finding all Hurwitz groups:

Proposition 1. If $X$ is a Mumford curve of genus $g \notin\{0,1,5,6,7,8\}$ with $F(g)$ automorphisms, then its genus is of the form $g=\left(p^{t}-1\right)^{2}$ for some integer $t$, its automorphism group $A$ is isomorphic to $\mathbf{Z}_{p}^{2 t} \rtimes D_{p^{t}-1}, A \backslash X=\mathbf{P}^{1}$, and $X \rightarrow A \backslash X$ is ramified above 3 points with ramification groups $\left(\mathbf{Z}_{2}, \mathbf{Z}_{2}, B(t, n)\right)$ if $p \neq 2$ and ramified above 2 points with groups $\left(\mathbf{Z}_{2}, B(t, n)\right)$ if $p=2$.

Proof. Let $*$ denote the amalgamation product. The computations in section 6 of [4] (cf. (6.9)-(6.12)) show the following:

Lemma. If $|A|=F(g)$, then, as abstract groups, $N \cong B(t, n) * \mathbf{z}_{n} D_{n}$ for $n=p^{t}-1$, and $g=\left(p^{t}-1\right)^{2}$. Furthermore, if $\gamma$ denotes an involution in $D_{n}-\mathbf{Z}_{n}, E$ denotes $\mathbf{Z}_{p}^{t}$ (seen as a normal subgroup of $\left.B(t, n)\right)$, and $E^{\prime}=\gamma E \gamma$, then $\bar{E} \cap \bar{E}^{\prime}=\{1\}$. 
Now observe that, since $\Gamma$ is free, any finite subgroup of $N$ is mapped by ${ }^{-}$to an isomorphic image. Also, the image of any relation in $N$ holds in $A$ - this applies, in particular, to the relations given by the semi-direct product structure in $B(t, n)$ and $D_{n}$. In the end, $A$ is a group of order $2 p^{2 t}\left(p^{t}-1\right)$ that contains the following subgroups: two elementary abelian groups $\bar{E}, \bar{E}^{\prime}$ that are disjoint, both of which are normalized by a cyclic group $\overline{\mathbf{Z}}_{n}$ of order $p^{t}-1$, which in its turn is normalized by an element $\bar{\gamma}$ of order 2 . Now $A$ is generated by the disjoint groups $\left\langle\bar{E}, \bar{E}^{\prime}, \overline{\mathbf{Z}}_{n}, \overline{\mathbf{Z}}_{2}\right\rangle$, and the order of $A$ equals the product of the orders of these groups. Hence $\bar{E}$ and $\bar{E}^{\prime}$ generate a subgroup of $A$ (namely, $\bar{E} \cdot \bar{E}^{\prime}$ ) of order $p^{2 t}$. Let $[\cdot, \cdot]$ denote the commutator. We will rely on the following group-theoretical lemma:

Lemma. Let $q=p^{t}$ be a power of a prime number, $G$ a group of order $q^{2}$, generated by two disjoint subgroups $\mathcal{E}, \mathcal{E}^{\prime}$ of order $q$. Assume that $\mathbf{Z}_{q-1}$ acts by automorphisms on $G$, stabilizing $\mathcal{E}$ and $\mathcal{E}^{\prime}$ such that the restriction of this action to $\mathcal{E}-\{1\}$ and $\mathcal{E}^{\prime}-\{1\}$ is simply transitive. Then $G=\mathcal{E} \times \mathcal{E}^{\prime}$.

Proof. Since $G$ is a $p$-group, it has a nontrivial center $Z$. Let $z \in Z$ be a nontrivial element. We claim that no element of $\mathbf{Z}_{q-1}$ fixes $z$ (we will write the action exponentially). Indeed, write $z=a b$ for $a \in \mathcal{E}$ and $b \in \mathcal{E}^{\prime}$. This way of writing is unique, since $G=\mathcal{E} \cdot \mathcal{E}^{\prime}$ and $\mathcal{E} \cap \mathcal{E}^{\prime}=\{1\}$. Let $\sigma \in \mathbf{Z}_{q-1}$; then, if $z^{\sigma}=z, a b=a^{\sigma} b^{\sigma}$, so $a=a^{\sigma}$ and $b=b^{\sigma}$ by the uniqueness of writing. However, since the cyclic group is assumed to act simply transitively on $\mathcal{E}-\{1\}$ and $\mathcal{E}^{\prime}-\{1\}$, this can only happen if $a=b=1$, so $z=1$, a contradiction.

Hence the set $\mathbf{Z}_{q-1} \cdot z$ has $q-1$ elements, and it belongs to $Z$, since $\mathbf{Z}_{q-1}$ acts by automorphisms on $G$. Since $Z$ is a subgroup of $G$, its order has to divide $q^{2}$; so it is at least $q$. Assume that $Z \cap \mathcal{E}=\{1\}$. Then $G=Z \cdot \mathcal{E}$, so $|Z|=q$, and since $Z$ is normal in $G$, it follows that $G=Z \rtimes \mathcal{E}$. So actually $G=Z \times \mathcal{E}$, contradicting the fact that $Z$ is the center of $G$.

Hence we can find a nontrivial element $\epsilon \in Z \cap \mathcal{E}$ so that $\left[\epsilon, \mathcal{E}^{\prime}\right]=1$. Acting on this with $\mathbf{Z}_{q-1}$ (which is transitive on $\mathcal{E}$ ) shows that $\left[\mathcal{E}, \mathcal{E}^{\prime}\right]=1$, so that $G=\mathcal{E} \times \mathcal{E}^{\prime}$ as desired.

This lemma implies immediately that $A=\left(\bar{E} \times \bar{E}^{\prime}\right) \rtimes \bar{D}_{n}$. Since the statement about $A \backslash X$ is in [4] (cf. (2.4)), the proof of Proposition 1 is finished.

Remark 2. From the above proof, we see that $\Gamma=\operatorname{ker}(\cdot)$ contains $[E, \gamma E \gamma]$, and actually has to equal it, since $\left(E * E^{\prime}\right) /\left[E, E^{\prime}\right]=E \times E^{\prime}$. Up to conjugation, one can embed $E$ into $P G L(2, k)$ only as upper triangular matrices $E=\left(\begin{array}{cc}1 & V \\ 0 & 1\end{array}\right)$ for some $\mathbf{F}_{p^{-}}$ vector space $V$ of dimension $t$ in $k$, and actually, since a cyclic group $\mathbf{Z}_{n}\left(n=p^{t}-1\right)$ has to act semidirectly on it, this cyclic $\mathbf{Z}_{n}$ has to be embedded as diagonal matrices over $\mathbf{F}_{p^{t}}^{*}$, and hence $V$ has to be of the form $\mathbf{F}_{p^{t}} \cdot x$ for some $x \in k^{*}$. We can assume $x=1$ by conjugating again with a suitable diagonal matrix. Then $\gamma$ can only be embedded as $\gamma=\left(\begin{array}{ll}0 & 1 \\ C & 0\end{array}\right)$ for some $C \in k^{*}$. Thus, up to $P G L(2, k)$-conjugation (viz., isomorphism of the corresponding Mumford curves, cf. [6], IV.3.10), the group $\Gamma$ is completely characterized by specifying the number $C \in k^{*}$, which should satisfy $|C|>1$ so that $\Gamma$ is indeed a discrete subgroup of $P G L(2, k)$ - this follows, e.g., from the method of isometric circles (cf. [4, section 8): the isometric circle of any element of $E$ is (in our normalization) the unit circle $\{|z|=1\}$, whereas the isometric circle of any element of $E^{\prime}$ is $\gamma \cdot\{|z|=1\}=\{|z-C|=1\}$. For $E * E^{\prime}$ to be discretely embedded in $P G L(2, k)$, these isometric circles should not intersect, leading to $|C|>1$. 
It would be interesting to find the relation (via automorphic forms?) between the parameter $c$ in the algebraic description $X_{t, c}$ and $C$ in this description of the Schottky group.

The theorem will now follow from the following algebraic fact:

Proposition 2. Let $X$ be an ordinary algebraic curve over a field $k$ of characteristic $p>0$ whose automorphism group $A$ is isomorphic to $\mathbf{Z}_{p}^{2 t} \rtimes D_{p^{t}-1}$. Assume that, for $p \neq 2$, the quotient by $A$ is of the form $X \rightarrow A \backslash X=\mathbf{P}^{1}$ and is ramified above 3 points, say, $\left(P_{1}, P_{2}, P_{3}\right)$ with ramification groups $\left(\mathbf{Z}_{2}, \mathbf{Z}_{2}, B(t, n)\right)$. If $p=2$, suppose that two points $\left(P_{1}, P_{2}\right)$ are ramified with groups $\left(\mathbf{Z}_{2}, B(t, n)\right)$. Then $X$ is isomorphic to an Artin-Schreier curve $X_{t, c}$ for some $c \in k^{*}$.

Proof. Let $p \neq 2$. The automorphism cover $X \rightarrow A \backslash X=\mathbf{P}^{1}$ has to decompose into a tower of successive Galois extensions as follows:

$$
X \rightarrow X_{1}:=\mathbf{Z}_{p}^{2 t} \backslash X \rightarrow X_{2}:=\mathbf{Z}_{n} \backslash X_{1} \rightarrow X_{3}:=\mathbf{Z}_{2} \backslash X_{2} .
$$

For this tower to have the correct ramification behaviour, the following should hold. In $X_{2} \rightarrow X_{3}$, exactly $P_{1}$ and $P_{2}$ should ramify; hence $X_{2}=\mathbf{P}^{1}$. Let $P_{3,1}$ and $P_{3,2}$ denote the points of $X_{2}$ above $P_{3}$. Since $X_{1} \rightarrow X_{2}$ is a tame cover in which only the two points $P_{3, i}$ should ramify, they have to ramify completely, and $X_{1}=\mathbf{P}^{1}$. Denote the points in $X$ above $P_{3, i}$ in $X_{1}$ by the same symbol. Now $X \rightarrow X_{1}=\mathbf{P}^{1}$ is an elementary abelian $p$-cover in which two points should ramify with ramification groups $\mathbf{Z}_{p}^{t}$. We can reorder the situation so that $X$ admits two quotients $X^{(1)}=E_{1} \backslash X$ and $X^{(2)}=E_{2} \backslash X$ for two groups $E_{1}=E_{2}=\mathbf{Z}_{p}^{t}$ such that $P_{3, i}$ branches completely in $X^{(i)}$ and $P_{3, j}$ is unbranched in $X^{(i)}$ for $i \neq j$. Let $z$ be a coordinate on $X_{1}=\mathbf{P}^{1}$, and set $z\left(P_{3,1}\right)=0$ and $z\left(P_{3,2}\right)=\infty$. The equation of $X^{(1)}$ has to be a succession of $p$-covers of $\mathbf{P}^{1}$ in which exactly one point ramifies completely, and since $X$ is ordinary, the conductor of these $p$-covers has a simple pole (compare [9]). By suitable normalization, $X^{(i)} \cong \mathbf{P}_{x_{i}}^{1} \rightarrow X_{1} \cong \mathbf{P}_{z}^{1}$ is given by an equation

$$
\prod_{\alpha \in V_{i}}\left(x_{i}-\alpha\right)=c_{i} z^{3-2 i} \quad(i=1,2)
$$


$x_{2}$ (corresponding to $X_{2} \rightarrow X_{3}$ ) should induce an isomorphism $X^{(1)} \rightarrow X^{(2)}$, we have $V_{1}=V_{2}$. Similarly, the existence of the automorphisms $x_{1} \mapsto \zeta x_{1}, x_{2} \mapsto \zeta^{-1} x_{2}$ for $\zeta \in \mathbf{F}_{p^{t}}^{*}$ (corresponding to $X_{1} \rightarrow X_{2}$ ) implies that $V_{1}=\mathbf{F}_{p^{t}} \cdot u$ for some $u \in k^{*}$. If we multiply $x_{1}, x_{2}$ and $z$ by this $u$, the equation of $X^{(1)}$ is $x_{1}^{p^{t}}-x_{1}=c_{1} z$, and that of $X^{(2)}$ is $x_{2}^{p^{t}}-x_{2}=\frac{c_{2}}{z}$. Finally, the fiber product $X^{(1)} \times_{X_{1}} X^{(2)}$ dominates $X$, and it has the same degree over $X_{1}$ as $X$; hence it equals $X$. But this fiber product is exactly equal to $X_{t, c}$, which finishes the proof for $p \neq 2$.

For $p=2$, except for the fact that $X_{2} \rightarrow X_{3}$ is Artin-Schreier of order two with a unique totally ramified point (instead of Kummer with two ramified points), the proof is entirely analogous.

Remark 3. If the moduli space of Mumford curves of genus $g$ over $k$ is stratified according to automorphism groups, then this proposition shows that the stratum with maximal automorphism group is exactly equal to the locus of Artin-SchreierMumford curves, and hence it is rigid-analytically connected. This connectedness statement can fail to hold for more general strata; see for example [7]. Nevertheless, 
the dimension of more general equivariant deformation spaces of Mumford curves can be computed in terms of the ramification data associated to the automorphism group (or the tree of groups associated with the normalizer of the Schottky group), cf. [3.

\section{REFERENCES}

[1] J. Cohen, Families of compact Riemann surfaces with automorphism groups, J. London Math. Soc. (2) 24 (1981), no. 1, 161-164. MR 82g:14031

[2] M. Conder, Hurwitz groups: a brief survey, Bull. Amer. Math. Soc. (N.S.) 23 (1990), no. 2, 359-370. MR 91d:20032

[3] G. Cornelissen and F. Kato, Equivariant deformation of Mumford curves and of ordinary curves in positive characteristic, Duke Math. J. 116 (2003), no. 3, 431-470.

[4] G. Cornelissen, F. Kato, and A. Kontogeorgis, Discontinuous groups in positive characteristic and automorphisms of Mumford curves, Math. Ann. 320 (2001), no. 1, 55-85. MR 2003d:14032

[5] G. Cornelissen and F. Kato, Mumford curves with maximal automorphism group II: Lamé type groups in genus 5-8, Geom. Dedicata 102 (2003), 127-142.

[6] L. Gerritzen and M. van der Put, Schottky groups and Mumford curves, Lecture Notes in Math., vol. 817, Springer-Verlag, Berlin - Heidelberg - New York, 1980. MR 82j:10053

[7] F. Kato, Mumford curves in a specialized pencil of sextics, Manuscripta Math. 104 (2001), no. 4, 451-458. MR 2002c: 14045

[8] D. Mumford, An analytic construction of degenerating curves over complete local rings, Compositio Math. 24 (1972), 129-174. MR 50:4592

[9] D. Subrao, The p-rank of Artin-Schreier curves, Manuscripta Math. 16 (1975), 169-193. MR 51:12868

Mathematisch Instituut, Universiteit Utrecht, Postbus 80.010, 3508 TA Utrecht, NEDERLAND

E-mail address: cornelis@math.uu.nl

Department of Mathematics, Faculty of Science, Kyoto University, Kyoto 606-8502, JAPAN

E-mail address: kato@math.kyoto-u.ac.jp 